\title{
Theoretical model of the nonlinear resonant interaction of whistler-mode waves and field-aligned electrons.
}

\author{
A. V. Artemyev, ${ }^{1, \text { a) }}$ A. I. Neishtadt, ${ }^{2, \text { b) }}$ J. M. Albert, ${ }^{3}$ L. Gan, ${ }^{4}$ W. $\mathrm{Li}{ }^{4}$ and Q. Ma ${ }^{4}$ \\ ${ }^{1)}$ Institute of Geophysics and Planetary Physics, University of California, Los Angeles, CA 90024, \\ USA. \\ ${ }^{2)}$ Department of Mathematical Sciences, Loughborough University, Loughborough LE11 3TU, \\ United Kingdom. \\ 3) Air Force Research Laboratory, Kirtland Air Force Base, Albuquerque, NM 87123, \\ $U S A$. \\ ${ }^{4)}$ Center for Space Physics, Boston University, Boston, MA 02215, USA.
}

(Dated: 9 April 2021)

The nonlinear resonant interaction of intense whistler-mode waves and energetic electrons in the Earth's radiation belts is traditionally described by theoretical models based on the consideration of slow-fast resonant systems. Such models reduce the electron dynamics around the resonance to the single pendulum equation, that provides solutions for the electron nonlinear scattering (phase bunching) and phase trapping. Applicability of this approach is limited to not-too-small electron pitch-angles (i.e., sufficiently large electron magnetic moments), whereas model predictions contradict to the test particle results for small pitch-angle electrons. This study is focused on such field-aligned (small pitch-angle) electron resonances. We show that the nonlinear resonant interaction can be described by the slow-fast Hamiltonian system with the separatrix crossing. For the first cyclotron resonance, this interaction results in the electron pitch-angle increase for all resonant electrons, contrast to the pitch-angle decrease predicted by the pendulum equation for scattered electrons. We derive the threshold value of the magnetic moment of the transition to a new regime of the nonlinear resonant interaction. For field-aligned electrons the proposed model provides the magnitude of magnetic moment changes in the nonlinear resonance. This model supplements existing models for not-toosmall pitch-angles and contributes to the theory of the nonlinear resonant electron interaction with intense whistler-mode waves.

\section{INTRODUCTION}

The wave-particle resonant interaction is the key process for energy exchange between different particle populations in collisionless plasma ${ }^{1}$. Particle scattering by waves is responsible for losses in magnetic traps ${ }^{2}$, e.g. in Earth's radiation belts ${ }^{3,4}$ where whistler-mode chorus and hiss waves together with electromagnetic ion cyclotron waves control electron precipitation into Earth's atmosphere $^{5-7}$. The basic concept describing such scattering is the quasi-linear theory ${ }^{8,9}$ that assumes electron resonant interaction with a broad-band spectrum of low coherence, low amplitude waves ${ }^{10}$. In an inhomogeneous ambient magnetic field the requirement for a low coherence is significantly relaxed ${ }^{11,12}$, and electron scattering by the monochromatic low amplitude waves can be described by the quasi-linear diffusion ${ }^{13,14}$. However, effects of electron resonances with intense waves, e.g. phase trapping and nonlinear scattering (phase bunching) ${ }^{15-20}$, are well beyond the quasi-linear theory and require a separate consideration ${ }^{21-24}$.

\footnotetext{
a) Also atSpace Research Institute of the Russian Academy of Sciences (IKI), 84/32 Profsoyuznaya Str, Moscow, 117997, Russia; Author to whom correspondence should be addressed; Electronic mail: aartemyev@igpp.ucla.edu

b) Also atSpace Research Institute of the Russian Academy of Sciences (IKI), 84/32 Profsoyuznaya Str, Moscow, 117997, Russia
}

Although oblique whistler-mode waves represent a significant fraction of whistlers in the Earth's radiation belts $^{25}$, the most intense are field-aligned whistlers ${ }^{26-28}$ (e.g., lower band chorus waves with the wave frequency $\omega$ below a half of the electron gyrofrequency, $\left.\Omega_{c e}\right)$, which often resonate with electrons nonlinearly ${ }^{29}$. There is only the first cyclotron resonance available for fieldaligned whistler-mode waves interacting with electrons: $\gamma \omega-k c \sqrt{\gamma^{2}-1} \cos \alpha=\Omega_{c e}$ ( $k$ is the wavevector, $\alpha$ is an electron pitch-angle, and $\gamma$ is an electron Lorentz factor). The phase trapping for this resonance results in electron acceleration with the electron pitch-angle increase for energetic particles (with the $\gamma<\Omega_{c e} / \omega$ ) and with the electron pitch-angle decrease for ultra-relativistic particles (with $\gamma>\Omega_{c e} / \omega$ ), see Refs. 30-32. The nonlinear scattering (phase bunching) results in decrease of electron energy and pitch-angle $\mathrm{e}^{33,34}$. Although effects of realistic wave frequency drift $^{35-39}$ and wave amplitude modulation ${ }^{40-43}$ alter the electron nonlinear resonant interaction, the basic concept remains the same: trapping results in electron transport away from the loss-cone and nonlinear scattering results in electron transport toward the loss-cone. A competition of these two nonlinear processes determines electron acceleration and losses.

The theory of nonlinear electron resonances with whistler-mode waves is based on individual orbit analysis, that reduces the electron motion equation to the pendulum equation with torque ${ }^{15,44-47}$. Such analysis describes well both phase trapping and nonlinear scat- 
tering effects and provides typical amplitudes of energy and pitch-angle changes, $\Delta \gamma$ and $\Delta \alpha$. The basic idea behind this analysis is the separation of time-scales of fast variations of resonant phase (the inverse time scale is $\sim \dot{\phi} \sim \omega$ ) and slow variations of the ambient magnetic fields along electron trajectories (the inverse timescale is $\sim c \sqrt{1-\gamma^{-2}} / R \ll \omega, R$ is a typical inhomogeneity scale). This separation provides a single small parameter $c / R \omega \sim 1 / k R \ll 1$. For the nonlinear waveparticle interaction this parameter is about the ratio of a wave amplitude $B_{w}$ and ambient magnetic field magnitude $B_{0}$, i.e. a wave force $\sim k B_{w}$ can compete with a mirror force $\sim B_{0} / R$ and temporally trap electrons into the resonance ${ }^{17,47,48}$. However, this theoretical concept is invalid for systems with the second small parameter, e.g. for very small pitch-angle (almost field-aligned) electrons. This effect has been found in Ref. 49: the resonant interaction cannot result in decrease of electron pitch-angles below zero, and for sufficiently small pitch-angles such interaction would increase pitch-angles. Therefore, the nonlinear scattering model, predicting $\Delta \alpha<0$, meets difficulties in describing small pitch-angle electron resonances (see discussion in Refs. 42 and 50). Test particle simulations show that $\Delta \alpha$ due to nonlinear resonant interaction becomes positive for all electrons with sufficiently small initial $\alpha$ (such electron repulsion from the loss-cone is the so-called anomalous electron trapping ${ }^{50}$ ). The similar effect of the absence of pitch-angle scattering with $\alpha$ decrease around the loss-cone is observed for the electron resonant interaction with electromagnetic ion cyclotron waves (see Refs. 51-53). So, the actual question is: can the theoretical model of the nonlinear wave-particle interaction be modified to account for such electron repulsion from the loss-cone? We address this question below.

\section{BASIC EQUATIONS}

We start with the Hamiltonian of a relativistic electron $\left(m_{e}\right.$ is the rest mass, $-e$ is the charge, energy is comparable to $m_{e} c^{2}$ where $c$ is the speed of light) describing two pairs of conjugate variables: the field-aligned coordinate and momentum $\left(s, p_{\|}\right)$, gyrophase $\psi$ and momentum $I_{x}=c \mu / e$ where $\mu$ is the classical magnetic moment. In presence of a field-aligned whistler-mode wave, this Hamiltonian can be written as (see, e.g., Refs. 23 and 54):

$$
\begin{aligned}
H & =m_{e} c^{2} \gamma+U_{w}\left(s, I_{x}\right) \sin (\phi+\psi) \\
\gamma & =\sqrt{1+\frac{p_{\|}^{2}}{m_{e}^{2} c^{2}}+\frac{2 I_{x} \Omega_{c e}}{m_{e} c^{2}}}
\end{aligned}
$$

where $\Omega_{c e}=e B_{0} / m_{e} c$ is the electron gyrofrequency $\left(B_{0}(s)\right.$ is the background magnetic field given by, e.g., reduced dipole $\left.\operatorname{model}^{44}\right), U_{w}=\sqrt{2 I_{x} \Omega_{c e} m_{e}} e B_{w} / \gamma m_{e} c k$ with $B_{w}$ the wave amplitude. The wave number $k(\omega, s)$ is given by the cold plasma dispersion ${ }^{55}$ for a constant wave frequency $\omega$ (i.e., $\partial \phi / \partial s=k, \partial \phi / \partial t=-\omega)$. Hamiltonian equations for (1) are

$$
\begin{aligned}
\dot{s} & =\frac{p_{\|}}{m_{e} \gamma}+\frac{\partial U_{w}}{\partial p_{\|}} \sin (\phi+\psi) \\
\dot{p}_{\|} & =-\frac{I_{x}}{\gamma} \frac{\partial \Omega_{c e}}{\partial s}-k U_{w} \cos (\phi+\psi)-\frac{\partial U_{w}}{\partial s} \sin (\phi+\psi) \\
\dot{\psi} & =\frac{\Omega_{c e}}{\gamma}+\frac{\partial U_{w}}{\partial I_{x}} \sin (\phi+\psi) \\
\dot{I}_{x} & =-U_{w} \cos (\phi+\psi)
\end{aligned}
$$

where $\dot{\phi}=k \dot{s}-\omega$. Equations (2) show that in absence of wave $\left(U_{w}=0\right)$ and for $I_{x}$ of the order of $p_{\|}^{2} / m_{e} \Omega_{c e}$ (for not too small pitch-angles), phases $\phi$ and $\psi$ change with the rate $\sim \Omega_{c e}$ (we consider whistler-mode waves with $\omega$ of the order of $\left.\Omega_{c e}\right)$, whereas $\left(s, p_{\|}\right)$change with the rate $\left(p_{\|} / \gamma\right) \partial \Omega_{c e} / \partial s \sim c / R$ where $R$ is a spatial scale of $B_{0}$ gradient (for the Earth radiation belts $R \approx R_{E} L$, $R_{E} \approx 6380 \mathrm{~km}$ and $L$ is the distance from the Earth in $\left.R_{E}\right)$. Comparing these rates, we obtain $c / R \Omega_{c e} \ll 1$, i.e., $\phi$ and $\psi$ change much faster than $\left(s, p_{\|}\right)$do. For intense whistler waves in the radiation belts $B_{w} / B_{0} \geq c / R \Omega_{c e}$ despite that $B_{w} / B_{0} \ll 1$ (see Ref. 27). Therefore, $\sim U_{w}$ term in Eqs. (2) does not modify the rates of $\phi, \psi$ and $\left(s, p_{\|}\right)$change: phases are fast variables and field-aligned coordinate, momentum are slow variables. Hamiltonian (1) with such time-scale separation has been studied both numerically and analytically ${ }^{34,41,45,47}$. Figure 1(a) shows several fragments of electron trajectories around the resonance $\dot{\phi}+\dot{\psi}=0$ for this Hamiltonian. Resonant electrons can be either trapped (and accelerated) by the wave or scattered (with energy decrease). Trapping increases $I_{x}$ (and increases electron equatorial pitch-angle $\left.\alpha_{e q} ; \quad I_{x}=m_{e} c^{2}\left(\gamma^{2}-1\right) \sin ^{2} \alpha_{e q} / 2 \Omega_{c e}(0)\right)$, whereas nonlinear scattering decreases $I_{x}$ and $\alpha_{e q}$. Amplitudes of energy and $I_{x}$ (or pitch-angle) changes for such trapping and scattering are well described by analytical equations ${ }^{33,54,56}$.

For electron nonlinear scattering, the amplitude of $I_{x}$ changes is about $\sim \sqrt{B_{w} / B_{0}}$ (see details in, e.g., Refs. 23,24 , and 57 and references therein). However, if $I_{x}$ is sufficiently small (i.e., for field-aligned electrons), these changes can be larger than the initial $I_{x}$. This would break the theory, because $I_{x}$ is a positively defined variable that cannot become negative. Figure 1(b) shows several fragments of field-aligned electron trajectories: there are only positive $I_{x}$ changes due to trapping and absence of scattering (compare with Fig. 1(a)). Thus, a new model should be developed to describe this regime of $100 \%$ trapping for small $I_{x} \leq \sqrt{B_{w} / B_{0}}$.

Let us introduce new phase $\varphi$ and conjugate momentum $I$ through the generating function $W=$ $\left(\int k(\tilde{s}) d \tilde{s}-\omega t+\psi\right) I+s P$ :

$$
\begin{aligned}
H_{I} & =-\omega I+m_{e} c^{2} \gamma+U_{w} \sin \varphi \\
\gamma & =\sqrt{1+\frac{(P+k I)^{2}}{m_{e}^{2} c^{2}}+\frac{2 I \Omega_{c e}}{m_{e} c^{2}}}
\end{aligned}
$$



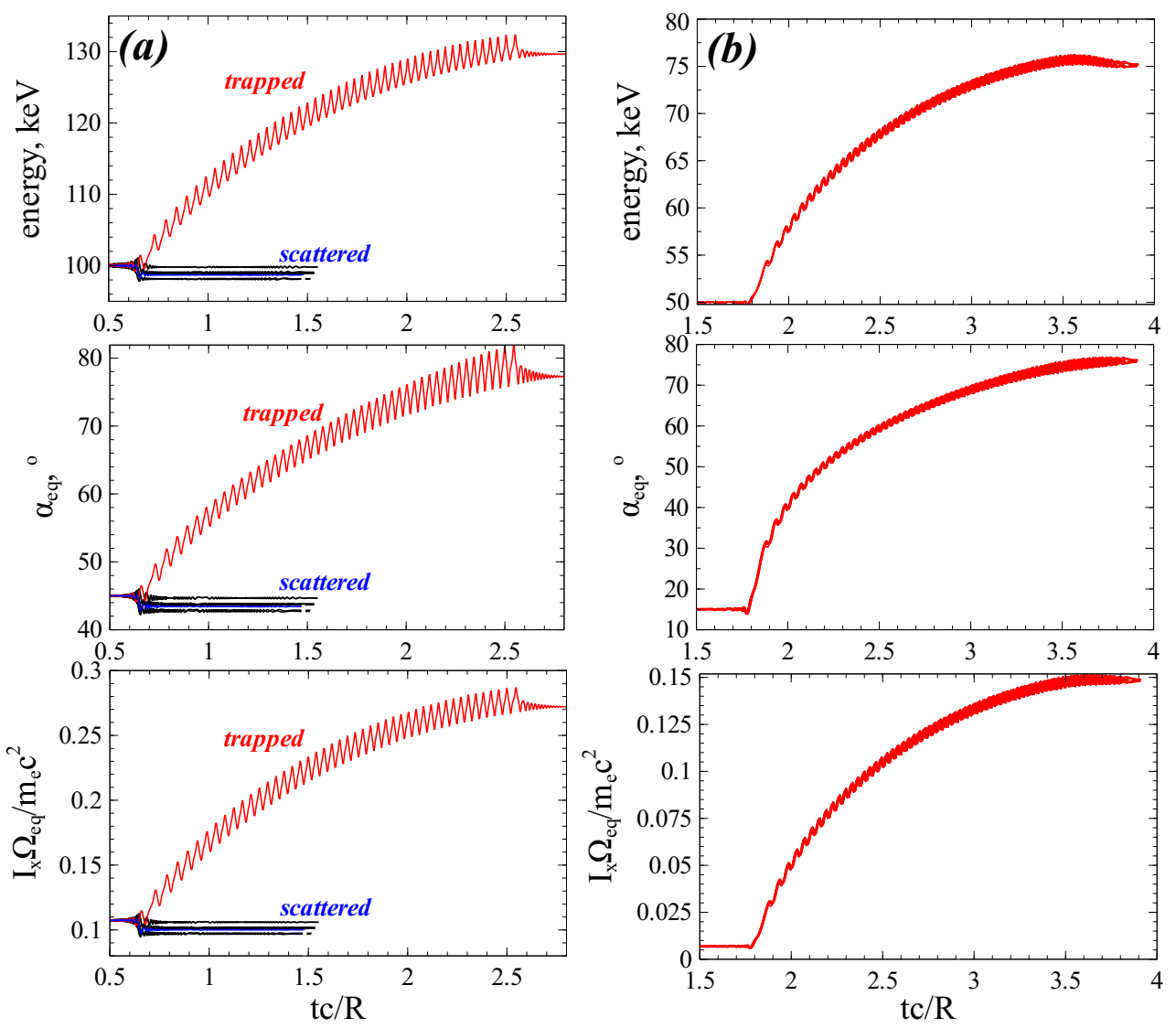

FIG. 1: Changes of electron energy, pitch-angle, and $I_{x}\left(\Omega_{e q}=\Omega_{c e}(0)\right.$ is the electron equatorial gyrofrequency) due to nonlinear scattering (black; blue shows averaged energy of scattered particles) and trapping (red). Left and right columns show results for $45^{\circ}$ and $15^{\circ}$ initial equatorial pitch-angles. The time interval of one resonant interaction is shown. For these trajectories we consider the curvature-free dipole magnetic field ${ }^{44}$ with the radial distance from the Earth $R=6 R_{E}$ (i.e., $L=6$ ). The wave frequency is $0.35 \Omega_{e q}$, and plasma frequency equals to $6 \Omega_{e q}$. To evaluate the wave number $k$ we use the cold plasma dispersion of whistler-mode waves ${ }^{55}$. Wave amplitude is 500 pT, i.e. this is an intense wave ${ }^{26,28,29,58}$. The distribution of the wave amplitude along magnetic field lines, $B_{w}(s)$, is modeled by

function $\tanh \left(\left(\lambda / \delta \lambda_{1}\right)^{2}\right) \exp \left(-\left(\lambda / \delta \lambda_{2}\right)^{2}\right)$ with $\lambda$ being the magnetic latitude $\left(d s=R d \lambda \sqrt{1+\sin ^{2} \lambda} \cos \lambda\right)$ and $\delta \lambda_{1}=2^{\circ}, \delta \lambda_{2}=20^{\circ}$. This function fits the observed whistler-mode wave intensity distribution ${ }^{59}$. To simplify the simulations, we consider waves only in one hemisphere, $B_{w}=0$ for $s<0$, and thus there is only one resonance within one bounce period. Waves are moving away from the equatorial plane, $s=0$, to large $s$, i.e. only $k>0$ are included.

Hamiltonian (3) does not depend on time, i.e. $H_{I}=$ const and $m_{e} c^{2} \gamma-\omega I$ is the integral of motion. Far from the resonance $\dot{\varphi}=\partial H_{I} / \partial I=0$, particles move in $2 \mathrm{D}$ surface formed by intersection of $H_{I}(s, P, I)=$ const and $I=$ const. The standard procedure suggests expansion of Hamiltonian (3) around the resonance $I=I_{\text {res }}$ defined by $\partial H_{I} / \partial I=0$. However, for small momentum $I$ such an approach is not applicable. To follow an alternative approach, we start with the expansion for small $I$ and then consider the resonance.

\section{EXPANSION AROUND SMALL $I$}

Let us consider Hamiltonian (3) for small $k I$ values:

$$
\begin{aligned}
H_{I} & \approx\left(\frac{\Omega_{c e}+k P / m_{e}}{\gamma_{0}}-\omega\right) I+\frac{K^{2} I^{2}}{2 m_{e}}+m_{e} c^{2} \gamma_{0} \\
& +\sqrt{\frac{2 I \Omega_{c e}}{m_{e}}} \frac{e B_{w}}{c k \gamma_{0}} \sin \varphi
\end{aligned}
$$

where

$$
K^{2}=\left.m_{e}^{2} c^{2} \frac{\partial^{2} \gamma}{\partial I^{2}}\right|_{I=0}=\frac{1}{\gamma_{0}^{3}}\left(k^{2}-2 \frac{k P \Omega_{c e}}{m_{e} c^{2}}-\frac{\Omega_{c e}^{2}}{c^{2}}\right)
$$

and $\gamma_{0}=\sqrt{1+\left(P / m_{e} c\right)^{2}}$. Note Eq. (4) shows that $H_{I} \approx m_{e} c^{2} \gamma_{0}+O(I)$ is the integral of motion. We rewrite 
Hamiltonian (4) as

$$
\begin{aligned}
H_{I} & =\Lambda+\frac{1}{2 m_{e}} K^{2}\left(I-I_{R}\right)^{2}+\sqrt{\frac{2 I \Omega_{c e}}{m_{e}}} \frac{e B_{w}}{c k \gamma_{0}} \sin \varphi \\
\Lambda & =m_{e} c^{2} \gamma_{0}-\frac{m_{e}}{2 K^{2}}\left(\omega-\frac{\Omega_{c e}+k P / m_{e}}{\gamma_{0}}\right)^{2} \\
I_{R} & =\frac{m_{e}}{K^{2}}\left(\omega-\frac{\Omega_{c e}+k P / m_{e}}{\gamma_{0}}\right)
\end{aligned}
$$

where $I_{R}=I_{\text {res }}$ is the resonant momentum, because $\partial H_{I} / \partial I=0$ has a solution $I=I_{R}$. The Hamiltonian equations for $I$ and $\varphi$ are

$$
\begin{aligned}
& \dot{I}=-\sqrt{2 I \Omega_{c e} m_{e}} \frac{B_{w} \Omega_{c e}}{B_{0} k \gamma_{0}} \cos \varphi \\
& \dot{\varphi}=\frac{1}{m_{e}} K^{2}\left(I-I_{R}\right)+\sqrt{\frac{\Omega_{c e} m_{e}}{2 I}} \frac{B_{w} \Omega_{c e}}{B_{0} k \gamma_{0}} \sin \varphi
\end{aligned}
$$

where coefficients depend on slowly changing $(s, P)$. In the second equation of Eq. (7) the term $\sim\left(B_{w} / \sqrt{I}\right) \sin \varphi$ is the Lorentz force of the wave field (i.e. a bunching force). For the standard consideration of the waveparticle resonant interaction ${ }^{21-24}$ this force is weaker than the generalized inertial force $\sim K^{2}\left(I-I_{R}\right)$, whereas we focus on systems with so small $I$, that $\sim B_{w} / \sqrt{I}$ becomes comparable to $\sim K^{2}\left(I-I_{R}\right)$. To compare these two terms we introduce a small parameter

$$
\varepsilon=\frac{e B_{w}}{m_{e} c^{2} k_{e q}}
$$

and functions

$$
u=\frac{k_{e q}}{k} \sqrt{\frac{\Omega_{c e}}{\Omega_{e q}}} \frac{1}{w \gamma_{0}}, \quad w=\frac{K^{2} c^{2}}{\Omega_{e q}^{2}}
$$

with $\Omega_{e q}=\Omega_{c e}(0), k_{e q}=k(0)$. Then we introduce new variable $Y=I \Omega_{e q} / m_{e} c^{2} \varepsilon^{\beta}, Y_{R}=I_{R} \Omega_{e q} / m_{e} c^{2} \varepsilon^{\beta}$,

$$
\tau=\Omega_{e q} \varepsilon^{\beta} \int^{t} w\left(t^{\prime}\right) d t^{\prime},
$$

(i.e., $\left(w(t) \Omega_{e q} \varepsilon^{\beta}\right) d / d \tau=d / d t$ and integration is along electron trajectories) and rewrite Eqs. (7) as

$$
\begin{aligned}
& \frac{d Y}{d \tau}=-\varepsilon^{1-3 \beta / 2} \sqrt{2 Y} u \cos \varphi \\
& \frac{d \varphi}{d \tau}=\left(Y-Y_{R}\right)+\frac{\varepsilon^{1-3 \beta / 2}}{\sqrt{2 Y}} u \sin \varphi
\end{aligned}
$$

Equations (8) are Hamiltonian equations for

$$
H_{Y}=\frac{1}{2}\left(Y-Y_{R}\right)^{2}+\varepsilon^{1-3 \beta / 2} \sqrt{2 Y} u \sin \varphi
$$

The $\beta$ parameter is controlled by smallness of $I$ (and $\left.I_{R}\right)$. If $\varepsilon^{1-3 \beta / 2} \ll 1$ (i.e., $\beta<2 / 3$ ), then $I$ is sufficiently large, the Hamiltonian system resembles the general system with small perturbations $\sim \sin \varphi$ and the standard approach is applicable for description of such system ${ }^{24,57}$. We are interested in system with sufficiently small $I$, such that the perturbation magnitude $\varepsilon^{1-3 \beta / 2} \sim 1$ (i.e. $\beta \sim 2 / 3)$ and Hamiltonian is

$$
H_{Y}=\frac{1}{2}\left(Y-Y_{R}\right)^{2}+\sqrt{2 Y} u \sin \varphi
$$

Hamiltonian (10) does not contain small parameters, but coefficients $u, Y_{R}$ depend on slow variables. To describe dynamics of Hamiltonian system (10), we introduce new variables $p=\sqrt{2 Y} \cos \varphi, q=\sqrt{2 Y} \sin \varphi$ with

$$
\frac{\partial q}{\partial \varphi} \frac{\partial p}{\partial Y}-\frac{\partial q}{\partial Y} \frac{\partial p}{\partial \varphi}=1
$$

Thus, $(q, p)$ are new canonical coordinate and momentum, and new Hamiltonian takes the form

$$
F=\frac{1}{2}\left(\frac{1}{2} p^{2}+\frac{1}{2} q^{2}-Y_{R}\right)^{2}+u q
$$

System with Hamiltinian (11) is called the second fundamental model for resonance, and the principal dynamics of this system has been described in Ref. 60 (see also Ref. 61). In application to the wave-particle resonant interaction this system has been considered in, e.g., Refs. 19 and 20 .

Let us consider a profile of Hamiltonian (11) on the axis $p=0$ : $U=F_{p=0}=(1 / 2)\left(q^{2} / 2-Y_{R}\right)^{2}+u q$. Equation determining extrema of $U(q)$ function is $d U / d q=$ $(1 / 2) q^{3}-Y_{R} q+u=0$, that can be rewritten as $(1 / 2) \tilde{q}^{3}-(2 / 3) \tilde{q}\left(Y_{R} / Y_{R}^{*}\right)+1=0$ with $\tilde{q}=q / u^{1 / 3}$ and $Y_{R}^{*}=(3 / 2) u^{2 / 3}$. Figure $2(\mathrm{a})$ shows that for $Y_{R}<Y_{R}^{*}$ there is only one extremum and for $Y_{R}>Y_{R}^{*}$ there are three extrema of $U(q)$. Therefore, the phase portrait of Hamiltonian (11) have two types shown in Fig 2(b): for $Y_{R}<Y_{R}^{*}$ there is only one O-point in the phase plane and phase trajectories rotate around this point, whereas for $Y_{R}>Y_{R}^{*}$ there are two O-points and one $\mathrm{X}$-point (saddle point), and two separatrices $\ell_{1,2}$ demarcate the phase portrait onto three domains $G_{\text {outer }}, G_{\text {inner }}, G_{\text {inter }}$ (see also Ref. 60 and 61 and examples in Refs. 19 and 20).

For constant $u, Y_{R}$ system (11) is integrable one, whereas for slowly changing $u, Y_{R}$ we can introduce an adiabatic invariant $I_{p}=(2 \pi)^{-1} \oint p d q$ (because all phase trajectories in the portrait shown in Fig. 2(b) are closed; see Ref. 62). In absence of separatrix (for $Y_{R}<$ $\left.Y_{R}^{*}\right), I_{p}$ would conserve with the exponential accuracy $\sim \exp \left(-\varepsilon^{-1 / 3}\right)$ where $\varepsilon^{1 / 3} \ll 1$ separates time-scales of $u, Y_{R}$ change ( $s, P$ change) and $p, q$ change $^{62,63}$. For conserved $I_{p}$ the system becomes integrable and $Y$ well before the resonance equals to $Y$ well after the resonance. Note $I_{p}=(2 \pi)^{-1} \oint p d q=2 Y(2 \pi)^{-1} \oint \cos ^{2} \varphi d \varphi=Y$ far from $\ell_{1,2}$ or in absence of $\ell_{1,2}$.

For phase trajectories crossing the separatrices in the phase portrait from Fig. 2(b), there is a change of $I_{p}$ (see Refs. 64-66). This change can be separated into 
so-called dynamical jump $\sim \varepsilon^{1 / 3} \ln \varepsilon^{1 / 3}$ and geometrical jump $\sim O(1)$ (see details in reviews 67 and 68). The geometrical jump is much larger than dynamical one (and effects of such jumps for application to the wave-particle resonant interaction in system (11) have been considered in, e.g., Refs. 19 and 20).

The separatrix crossing results in particle transition from the region with area $2 \pi I_{p, \text { init }}\left(I_{p, \text { init }}\right.$ is the initial value of $I_{p}$ ) to the region with another area $S$. At the moment of the separatrix crossing the invariant $I_{p}$ becomes equal to $S / 2 \pi$. Thus, there is a jump $\Delta I_{p}=\Delta S / 2 \pi=S / 2 \pi-I_{p, \text { init }}$. This jump directly relates to the jump of $I_{x}$ of the initial system (1)

$$
\Delta I_{x}=\Delta I=\frac{m_{e} c^{2} \Delta Y}{\Omega_{e q} \varepsilon^{-2 / 3}}=\frac{m_{e} c^{2} \Delta S}{2 \pi \Omega_{e q}}\left(\frac{e B_{w}}{m_{e} c^{2} k_{e q}}\right)^{2 / 3}
$$

Therefore, to describe electron resonances in system (1) with small $I_{x}$ (small $I$ ), we should describe $I_{p}$ change due to separatrix crossing on the phase portrait shown in Fig. 2(b).

\section{DYNAMICS OF SYSTEM (11)}

Let us study properties of Hamiltonian (11) that are important for evaluation of the $\Delta I_{p}$ jump. First, we are interested in areas of regions $G_{\text {inner }}, G_{\text {inter }}, G_{\text {outer }}$. Coordinates of X-point $C$ in the $(q, p)$ plane are $\left(q_{c}, 0\right)$ where $q_{c}$ is the maximum root of equation $d U / d q=$ $(1 / 2) q^{3}-Y_{R} q+u=0$. We introduce polar coordinates $(\rho, \eta)$ as $q=q_{c}+\rho \cos \eta, p=\rho \sin \eta$ and rewrite Hamiltonian (11) as

$$
\begin{aligned}
F & =\frac{1}{2}\left(\frac{1}{2} \rho^{2}+\frac{1}{2} q_{c}^{2}-Y_{R}\right)^{2}+\frac{1}{2} q_{c}^{2} \rho^{2} \cos ^{2} \eta \\
& +\frac{1}{2} \rho^{3} q_{c} \cos \eta+u q_{c}
\end{aligned}
$$

Then, separatrices $\ell_{1,2}$ are defined by equation $F=F_{\rho=0}$ :

$$
\rho^{2}+4 \rho q_{c} \cos \eta+4 q_{c}^{2} \cos ^{2} \eta+4\left(\frac{q_{c}^{2}}{2}-Y_{R}\right)=0
$$

with the solution

$$
\rho_{ \pm}=-2 q_{c} \cos \eta \pm 2 \sqrt{Y_{R}-\frac{1}{2} q_{c}^{2}}
$$

Areas of $G_{i n n e r}, G_{\text {inter }}$, and $G_{\text {outer }}$ are given by equations:

$$
\begin{aligned}
S_{\text {inner }} & =\frac{1}{2} \oint \rho_{-}^{2} d \eta=\int_{\eta_{-}}^{\pi} \rho_{-}^{2} d \eta=4 Y_{R} \eta_{c}-3 q_{c}^{2} \sin 2 \eta_{c} \\
S_{\text {outer }} & =\frac{1}{2} \oint \rho_{+}^{2} d \eta=\int_{\eta_{+}}^{\pi} \rho_{+}^{2} d \eta=4 Y_{R}\left(\pi-\eta_{c}\right)+3 q_{c}^{2} \sin 2 \eta_{c} \\
S_{\text {inter }} & =\frac{1}{2} \oint\left(\rho_{+}^{2}-\rho_{-}^{2}\right) d \eta=S_{\text {outer }}-S_{\text {inner }} \\
& =4 Y_{R}\left(\pi-2 \eta_{c}\right)+6 q_{c}^{2} \sin 2 \eta_{c}
\end{aligned}
$$

where

$$
\begin{aligned}
& \int_{\eta_{ \pm}}^{\pi} \rho_{ \pm}^{2} d \tilde{\eta}=4 q_{c}^{2} \int_{\eta_{ \pm}}^{\pi} \cos ^{2} \eta d \tilde{\eta}-4\left(\frac{1}{2} q_{c}^{2}-Y_{R}\right)\left(\pi-\eta_{ \pm}\right) \\
\mp & 8 q_{c} \sqrt{Y_{R}-\frac{1}{2} q_{c}^{2}} \int_{\eta_{ \pm}}^{\pi} \cos \tilde{\eta} d \tilde{\eta}=4 Y_{R}\left(\pi-\eta_{ \pm}\right)-q_{c}^{2} \sin 2 \eta_{ \pm} \\
\pm & 8 q_{c} \sqrt{Y_{R}-\frac{1}{2} q_{c}^{2}} \sin \eta_{ \pm}=4 Y_{R}\left(\pi-\eta_{ \pm}\right)+3 q_{c}^{2} \sin 2 \eta_{ \pm} \\
= & \left\{\begin{array}{cc}
4 Y_{R}\left(\pi-\eta_{c}\right)+3 q_{c}^{2} \sin 2 \eta_{c} & \eta_{+}=\eta_{c} \\
4 Y_{R} \eta_{c}-3 q_{c}^{2} \sin 2 \eta_{c} & \eta_{-}=\pi-\eta_{c}
\end{array}\right.
\end{aligned}
$$

and $q_{c} \cos \eta_{c}=\sqrt{Y_{R}-q_{c}^{2} / 2}$ (solution of $\rho_{+}=0$, i.e. we take $\eta_{c}<\pi / 2$, see Fig. 3(a)).

Introducing $\tilde{q}_{c}=q_{c} / u^{1 / 3}$ and $y_{R}=Y_{R} / Y_{R}^{*}$ (where $\left.Y_{R}^{*}=(3 / 2) u^{2 / 3}\right)$, we rewrite Eqs. (16) as

$$
\begin{aligned}
S_{\text {inner }} & =\tilde{S}_{\text {inner }} 4 Y_{R}^{*}, \quad \tilde{S}_{\text {inner }}=y_{R} \eta_{c}-\tilde{q}_{c}^{2} \sin \eta_{c} \cos \eta_{c} \\
S_{\text {outer }} & =\tilde{S}_{\text {outer }} 4 Y_{R}^{*}, \quad \tilde{S}_{\text {outer }}=y_{R}\left(\pi-\eta_{c}\right)+\tilde{q}_{c}^{2} \sin \eta_{c} \cos \eta_{c} \\
S_{\text {inter }} & =\tilde{S}_{\text {inter }} 4 Y_{R}^{*}, \quad \tilde{S}_{\text {inter }}=y_{R}\left(\pi-2 \eta_{c}\right)+\tilde{q}_{c}^{2} \sin 2 \eta_{c} \\
\eta_{c} & =\operatorname{arcos} \sqrt{\frac{3}{2} \frac{y_{R}}{\tilde{q}_{c}^{2}}-\frac{1}{2}}=\operatorname{arcos}\left(\tilde{q}_{c}^{-3 / 2}\right)
\end{aligned}
$$

and $\tilde{q}_{c}^{3}-3 \tilde{q}_{c} y_{R}+2=0$. Figures $3(\mathrm{~b}, \mathrm{c})$ show $\tilde{q}_{c}, \cos \left(\eta_{c}\right)$, and areas $\tilde{S}_{\text {inner }}, \tilde{S}_{\text {outer }}, \tilde{S}_{\text {inter }}$ as function of $y_{R}$ parameter.

\section{ELECTRON DYNAMICS}

Figure $3(\mathrm{c})$ shows that areas $S_{\text {inner }}, S_{\text {outer }}, S_{\text {inter }}$ evolve with $y_{R}=Y_{R} / Y_{R}^{*}$, but $y_{R}$ is the function of slow time (slow variables) and evolves along the electron trajectory:

$$
\begin{aligned}
y_{R} & =\frac{Y_{R}}{Y_{R}^{*}}=\frac{2}{3} \frac{I_{R} \Omega_{e q}}{m_{e} c^{2}(u \varepsilon)^{2 / 3}} \\
& =\frac{2}{3} \frac{\kappa^{2 / 3} k^{2} c^{2}}{\gamma_{0}^{4 / 3} \Omega_{c e}^{2}} \frac{I_{R} \Omega_{c e}}{m_{e} c^{2}\left(B_{w} / B\right)^{2 / 3}}
\end{aligned}
$$

where

$$
\begin{aligned}
\frac{K^{2} c^{2}}{\Omega_{c e}^{2}} & =\frac{\kappa}{\gamma_{0}^{3}} \frac{k^{2} c^{2}}{\Omega_{c e}^{2}} ; \kappa=1-\frac{\Omega_{c e}^{2}}{k^{2} c^{2}}-2 \frac{\Omega_{c e}}{k c} \frac{P}{m_{e} c} \\
& =\gamma_{0}^{2}-\left(\frac{\Omega_{c e}}{k c}+\sqrt{\gamma_{0}^{2}-1}\right)^{2} \\
(u \varepsilon)^{2 / 3} & =\frac{\gamma_{0}^{4 / 3}}{\kappa^{2 / 3}}\left(\frac{\Omega_{c e}^{2}}{k^{2} c^{2}} \frac{e B_{w}}{m_{e} c^{2} k}\right)^{2 / 3} \frac{\Omega_{e q}}{\Omega_{c e}}
\end{aligned}
$$



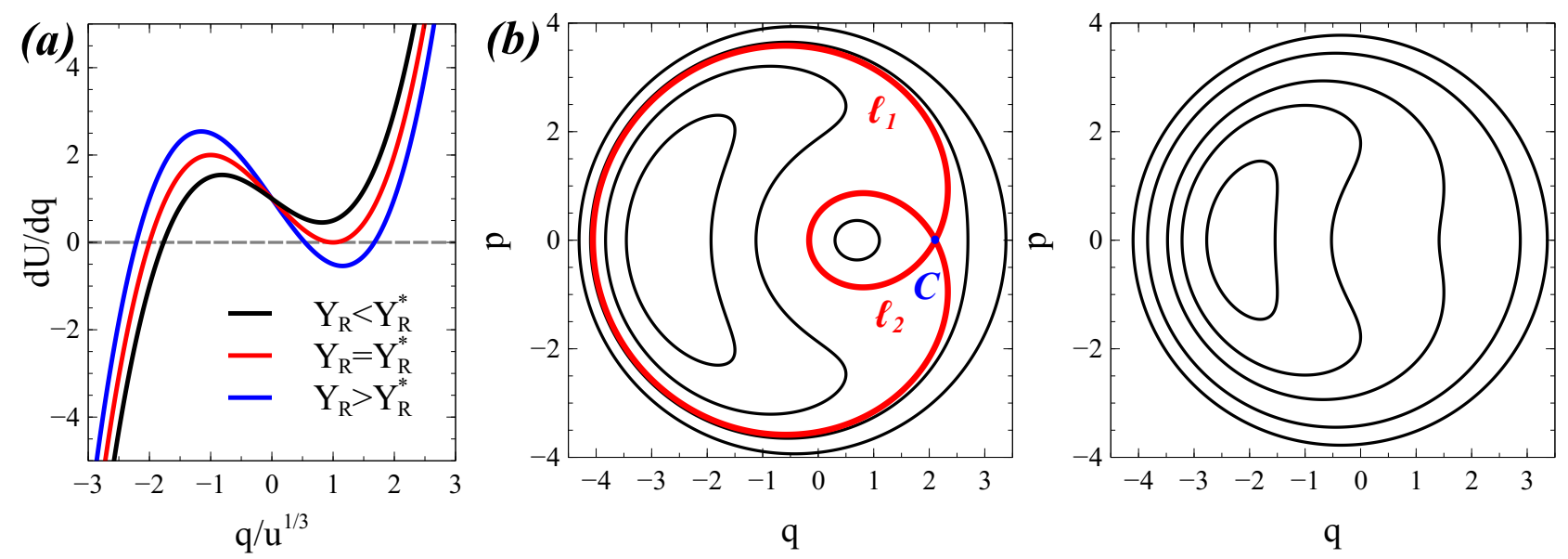

FIG. 2: (a) Profiles of $d U / d q=\tilde{q}^{3} / 2-(3 / 2) \tilde{q}\left(Y_{R} / Y_{R}^{*}\right)+1$ for different $Y_{R} ; Y_{R}^{*}=(3 / 2) u^{2 / 3}$ and $\tilde{q}=q / u^{1 / 3}$. (b) Phase portraits of system (11) with $u=2$ for $Y_{R}>Y_{R}^{*}$ (left) and $Y_{R}<Y_{R}^{*}$ (right). Bold red curve shows the separatrices $\ell_{1,2}$.
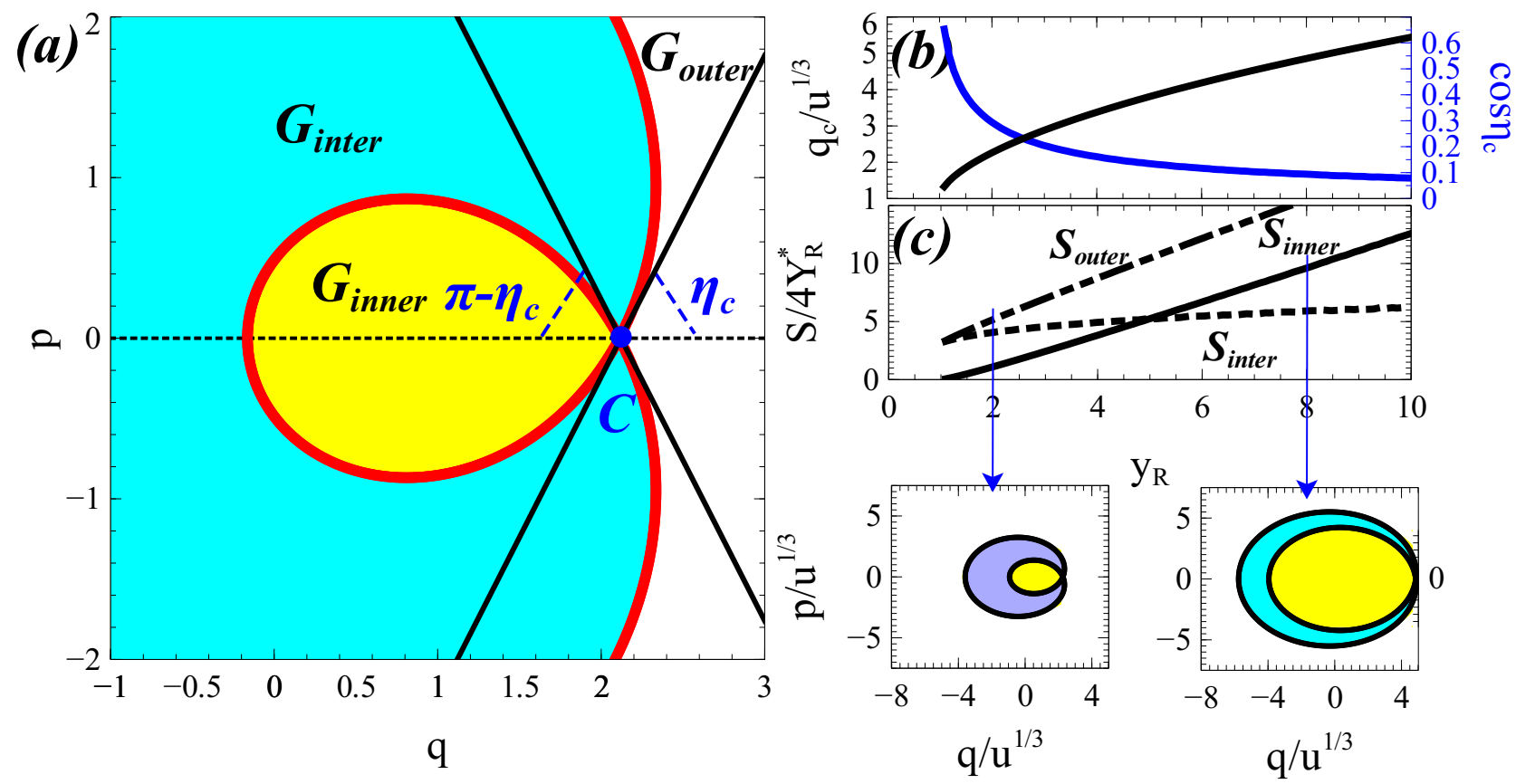

FIG. 3: (a) Schematic of $G_{\text {inner }}, G_{\text {inter }}$, and $G_{\text {outer }}$ regions; angle $\eta_{c}$ is shown. (b) and (c) Profiles of $\tilde{q}_{c}, \cos \eta_{c}$, $\tilde{S}_{\text {inner }}=S_{\text {inner }} / 4 Y_{R}^{*}, \tilde{S}_{\text {outer }}=S_{\text {outer }} / 4 Y_{R}^{*}$, and $\tilde{S}_{\text {inter }}=S_{\text {inter }} / 4 Y_{R}^{*}$; bottom panels show phase portraits for two $y_{R}=Y_{R} / Y_{R}^{*}$ values.

The resonant interaction starts with $I_{R}=I_{x}$ (initial $I$ value is $I_{x}$ ), and for this moment we can write

$$
\begin{aligned}
y_{R} & =\frac{2}{3} \frac{\kappa^{2 / 3} k^{2} c^{2}}{\gamma_{0}^{4 / 3} \Omega_{c e}^{2}} \frac{I_{x} \Omega_{c e}}{m_{e} c^{2}\left(B_{w} / B\right)^{2 / 3}} \\
\kappa & =\gamma_{0}^{2}\left(1-(\omega / k c)^{2}\right)
\end{aligned}
$$

The factor $\sim 2 I_{x} \Omega_{c e} / m_{e} c^{2}\left(B_{w} / B\right)^{2 / 3}$ is of the order of one due to smallness of $I_{x}$. In the resonance $y_{R}$ variation is described by Eq. (18) with $I_{R}$ given by Eq. (6).

Figure 4(a) shows $y_{R}$ variation with the magnetic latitude for particles moving from high latitudes toward the equatorial plane. Along the resonant trajectories $y_{R}$ increases, i.e. normalized areas $\tilde{S}_{\text {inner }}, \tilde{S}_{\text {outer }}$, and $\tilde{S}_{\text {inter }}$ should grow (see Fig. 3(c)). However, expression of $S=4 Y_{R}^{*} \tilde{S}$ includes the wave amplitude $u\left(Y_{R}^{*} \sim u^{2 / 3}\right)$ that generally drops to zero at the equatorial plane. Thus, $S$ dynamics along the resonant trajectories is determined by the competition of $\tilde{S}$ increase and $Y_{R}^{*}$ de- 
crease. Figure 4(b) shows that $S_{\text {inter }}$ decreases, whereas $S_{\text {inner }}$ increases. Taking into account that $y_{R}$ grows from values smaller than one (i.e. from values for which $S_{\text {inner }}=0$ and there is only $S_{\text {outer }}$; see Fig. $\left.3(\mathrm{c})\right)$, we describe resonant particle dynamics.

Far from the resonance, $Y$ equals to $\Omega_{e q} I_{x} / m_{e} c^{2} \varepsilon^{2 / 3}$ and equals to $I_{p}=(2 \pi)^{-1} \oint p d q$ for Hamiltonian system (11). Thus, for given $I_{x}$, in the $(p, q)$ plane electrons are distributed along the circle with the radius $\sqrt{2 Y}$; the entire circle is inside the region $G_{\text {outer }}$, because $G_{\text {inner }}$ and $G_{\text {inter }}$ do not exist (see schematic in Fig. 4(c), moment $\# 1$ ). With time (or with $y_{R}$ increase) regions $G_{\text {inner }}$, $G_{\text {inter }}$ form (when $y_{R}$ exceeds one), whereas trajectories on the $(q, p)$ plane deform, but save their areas. Then two scenarios are possible.

\section{A. Scenario for small $Y$}

If initial $Y$ is sufficiently small, at the moment of $G_{\text {inner,inter }}$ formation trajectories appear inside $G_{\text {inter }}$ (Fig. 4(c), moment \#2 shows such red trajectory within $\left.G_{\text {inte }}\right)$. From this moment and up to the sepatrix crossing the particle is in the resonance with the wave. When decreasing $S_{\text {inter }} / 2 \pi$ reaches $I_{p}$, electrons cross the separatrix $\ell_{2}$ and appear in $G_{\text {inner }}$ region $\left(S_{\text {outer }}\right.$ increases with $S_{\text {inter }}$, and electrons cannot come to $\left.G_{\text {outer }}\right)$ with growing $S_{\text {inner }}$ (see schematic in Fig. 4(c), moment \#3). This separatrix crossing results in a jump of the adiabatic invariant $\Delta I_{p}=\left(S_{\text {inner }}-S_{\text {inter }}\right) / 2 \pi$ where $S_{\text {inner }}$ is evaluated at the moment when $S_{\text {inter }}=2 \pi I_{p}$ (escape from the resonance). A new $I_{p}$ would be conserved, and far from the separatrix crossing (far from the resonance) new $Y$ is equal to new $I_{p}$. Thus, the jump of $Y$ equals to $\Delta Y=\left(S_{\text {inner }}-S_{\text {inter }}\right) / 2 \pi=4 Y_{R}^{*}\left(\tilde{S}_{\text {inner }}-\tilde{S}_{\text {inter }}\right) / 2 \pi$ where $Y_{R}^{*}$ is evaluated at the moment of the separatrix crossing (that can be quite far from the resonance when $G_{\text {inter }}$ forms). Jump $\Delta Y$ can be rewritten as a jump of $I_{x}$, see Eq. (13):

$$
\begin{aligned}
\Delta I_{x} & =\Delta\left\{\frac{\tilde{S}}{2 \pi} \frac{4 Y_{R}^{*} m_{e} c^{2} \varepsilon^{2 / 3}}{\Omega_{e q}}\right\}=\Delta\left\{\frac{3 \Delta \tilde{S}}{\pi} \frac{m_{e} c^{2}}{\Omega_{e q}}(u \varepsilon)^{2 / 3}\right\} \\
& =\Delta\left\{\frac{3 \tilde{S}}{\pi} \frac{m_{e} c^{2}}{\Omega_{c e}} \frac{\gamma_{0}^{4 / 3} \Omega_{c e}^{2}}{\kappa^{2 / 3} k^{2} c^{2}}\left(B_{w} / B\right)^{2 / 3}\right\}
\end{aligned}
$$

where all variables changing along magnetic field line should be evaluated at the separatrix crossing moment.

\section{B. Scenario for large $Y$}

If initial $Y$ is sufficiently large, then at the moment of $G_{\text {inner,inter }}$ formation the electron trajectories in the $(q, p)$ plane appear in $G_{\text {outer }}$. For such trajectories the separatrix crossing would results in $\Delta Y=\left(S_{\text {inner }}-\right.$ $\left.S_{\text {outer }}\right) / 2 \pi$. Because $S_{\text {outer }}>S_{\text {inner }}$, this $Y$ change (and
$I_{x}$ change) would mean $I_{x}$ decrease, i.e. this is classical nonlinear electron scattering on the resonance ${ }^{22-24}$.

\section{Electron drift in pitch-angle space}

Let us summarize results of $I_{p}(Y)$ changes. The separatrix crossing by trajectory in the $(q, p)$ plane occurs when $S_{\text {inter }}=2 \pi I_{p}$, and thus the moment of crossing depends on $I_{p}$ (i.e. on the initial $Y$ or, equivalently, on initial $I_{x}$ ). There are two possible situations shown in Fig. 5.

If the initial $I_{x}$ (initial $I_{p}=S_{\text {inter }} / 2 \pi$ ) is sufficiently small, then the separatrix crossing occurs when electron orbits are in $G_{i n t e r}$ and $S_{i n t e r}<S_{i n n e r}$ (this moment is quite far from the moment of $G_{\text {inter }}$ forming, i.e., particles spend a significant time $\sim O\left(\varepsilon^{1 / 3}\right)$ in the resonance before the separatrix crossing). Thus, jump $\Delta I_{p}=\left(S_{\text {inner }}-S_{\text {inter }}\right) / 2 \pi>0$ and $I_{x}$ increases due to the electron resonant interaction with the waves (see Fig. 5(a) and black trajectories in Fig. 1, right panels). Magnitude of $I_{x}$ change is defined by Eq. (22).

If the initial $I_{x}$ (initial $I_{p}$ ) is sufficiently large, then the separatrix crossing occurs when electron orbits are in $G_{\text {outer }}$. This happens right around the resonance, i.e. particles do not spend a long time being trapped in $G_{\text {outer }}$. Thus, jump $\Delta I_{p}=\left(S_{\text {inner }}-S_{\text {outer }}\right) / 2 \pi<0$ (because $S_{\text {outer }}>S_{\text {inner }}$ ) and $I_{x}$ decreases due to the electron resonant interaction with the waves (see Fig. 5(b) and black trajectories in Fig. 1, left panels).

These two situations explain classical scattering with $I_{x}$ decrease for electrons having not-too-small $I_{x}$ (see Refs. 22 and 23) and anomalous trapping with $I_{x}$ increase for all electrons with initial very small $I_{x}$ (see Refs. 42, 49, and 50).

\section{DISCUSSION}

Whistler-mode waves nonlinearly resonate with fieldaligned ( $\left.\operatorname{small}-I_{x}\right)$ electrons and transport them away from the loss-cone ${ }^{49}$, and this effect is called anomalous electron trapping ${ }^{50}$, when $100 \%$ of resonant electrons are phase trapped. Such trapping of field-aligned electrons differs significantly from whistler-mode wave interaction with moderate pitch-angle electrons, that results in scattering toward the loss-cone of the majority of resonant electrons.

Similar effect of the change of trapping/scattering relation has been found for electromagnetic ion cyclotron waves. These waves nonlinearly scatter electrons away from the loss-cone, whereas electron phase trapping results in pitch-angle decrease. ${ }^{52,53,69}$. For field-aligned ( $\operatorname{small}-I_{x}$ ) electrons the trapping disappears and there is only nonlinear scattering with the pitch-angle increase (see detailed analysis in Ref. 70, and references therein). As we show in this study, such modification of the nonlinear resonant interaction for small $I_{x}$ can be described an- 

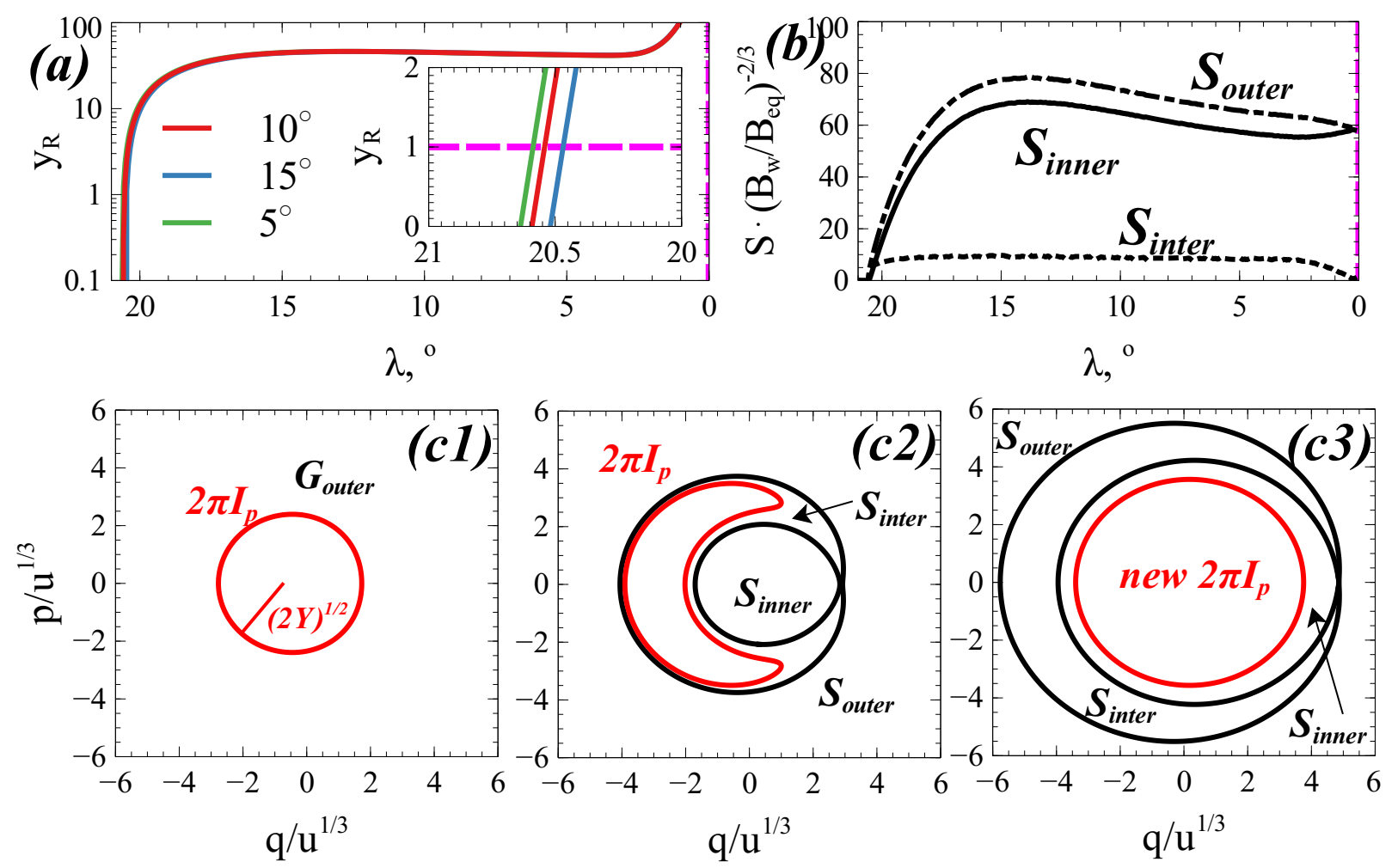

FIG. 4: (a) $y_{R}$ as a function of magnetic latitude for $50 \mathrm{keV}$ energy and three pitch-angles. (b) Areas

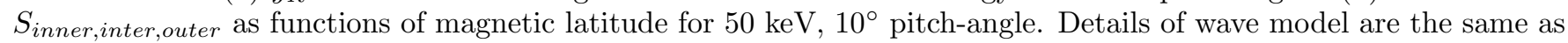
in the caption of Fig.1. (c) three schematic views of particle trajectories in the $(q, p)$ plane. Phase portraits change from c1 to c3 along particle trajectory.
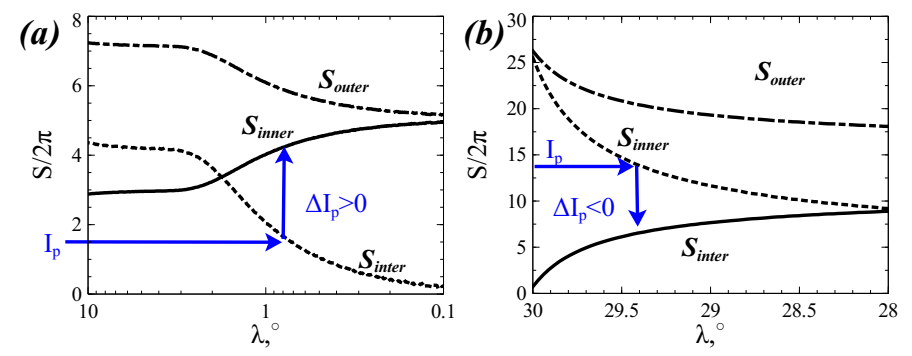

FIG. 5: Schematic of $I_{p}$ jumps due to separatrix crossing. (a) System with positive $\Delta I_{p}$; (b) System with negative $\Delta I_{p}$. For simplicity we use $I_{R} \approx I_{x}$ here.

alytically, because even for small $I_{x}$ there is a separation of time-scales of $(\varphi, I)$ variations and $\left(s, p_{\|}\right)$variations. Let us discuss and summarize obtained results.

\section{A. Electron dynamics around loss-cone}

Figure 5 shows that for some initial $I_{x}=I_{x}^{*}$, the separatrix crossing occurs with $\Delta I_{x}=0$, i.e. $S_{\text {inter }} \approx S_{\text {inner }}$. Thus, electrons with this $I_{x}^{*}$ would not experience pitchangle change (in the leading approximation). Let us consider a realistic system with $I_{x}^{*} \sim \varepsilon^{2 / 3}$ sufficiently small to keep derived equations valid. Change of $I_{x}$ can be written as $\Delta I_{x}=\Delta \alpha_{e q} \sin \alpha_{e q} \cos \alpha_{e q} m_{e} c^{2}\left(\gamma^{2}-\right.$ 1) $/\left(\Omega_{e q}-\sin ^{2} \alpha_{e q} \omega \gamma\right)$ where $\alpha_{e q}$ is the electron equatorial pitch-angle. We denote $\alpha_{e q}^{*}$ value of pitch-angle for which $I_{x}=I_{x}^{*}$ (for given energy $\gamma$ ), and write $\Delta \alpha_{e q}=$ $2 \Delta I_{x} \Omega_{e q} / m_{e} c^{2} \sin 2 \alpha_{e q} \approx\left(\alpha_{e q}-\alpha_{e q}^{*}\right) \nu^{\prime}$ where $\nu^{\prime}=$ $\partial \nu /\left.\partial \alpha_{e q}\right|_{\alpha_{e q}=\alpha_{e q}^{*}}$ and $\nu=2 \Delta I_{x}\left(\alpha_{e q}\right) \Omega_{e q} / m_{e} c^{2} \sin 2 \alpha_{e q}$ with $\nu\left(\alpha_{e q}^{*}\right)=0$.

Figure 6 shows two possible variants of field-aligned (small $I_{x}$ ) electron resonant interaction. Independently of $\nu^{\prime}$, electrons with $\alpha_{e q}<\alpha_{e q}^{*}$ experience the pitch-angle increase, i.e. transported away from the loss-cone $\alpha_{L C}$. Therefore, the transport into loss-cone (electron precipitations) would require that electrons with $\alpha_{e q}>\alpha_{e q}^{*}$ are scattered with sufficiently large $\Delta \alpha_{e q}: \alpha_{e q}+\Delta \alpha_{e q}<\alpha_{L C}$. If $\nu^{\prime}<-1, \alpha_{e q}+\Delta \alpha_{e q}<\alpha_{L C}$ can be rewritten as $\alpha_{e q} \geq\left(\left|\nu^{\prime}\right| \alpha_{e q}^{*}-\alpha_{L C}\right) /\left(\left|\nu^{\prime}\right|-1\right)$, and this inequality is always satisfied for $\alpha_{e q}>\alpha_{e q}^{*}$ and sufficiently small loss-cone $\alpha_{L C}<1$. Thus, for $\nu^{\prime}<-1$ there are electron nonlinear scattering into loss-cone and precipitations. If $\nu^{\prime}>-1, \alpha_{e q}+\Delta \alpha_{e q}<\alpha_{L C}$ can be rewritten as $\alpha_{e q} \leq\left(\alpha_{L C}-\left|\nu^{\prime}\right| \alpha_{e q}^{*}\right) /\left(1-\left|\nu^{\prime}\right|\right)$, and this inequality is satisfied for $\alpha_{e q}>\alpha_{e q}^{*}$ only for the exotic case $\alpha_{L C}>\alpha_{e q}^{*}$. Thus, if $\nu^{\prime}>-1$ there are no electron scattering into loss-cone and no precipitations (this is anomalous electron trapping, see Ref. 50). These estimates demon- 

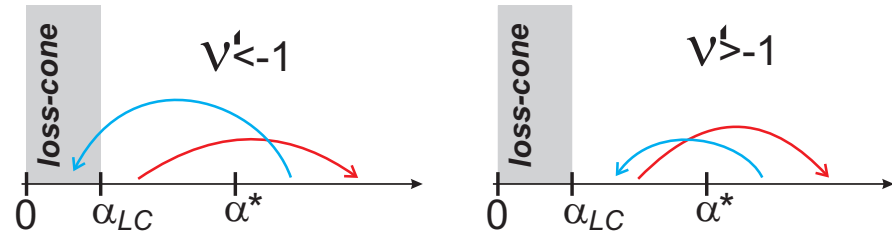

FIG. 6: Schematic view of pitch-angle jumps for system with electron losses (left panel, $\nu^{\prime}<-1$ ) and system without electron losses (right panel, $\nu^{\prime}>-1$ ).
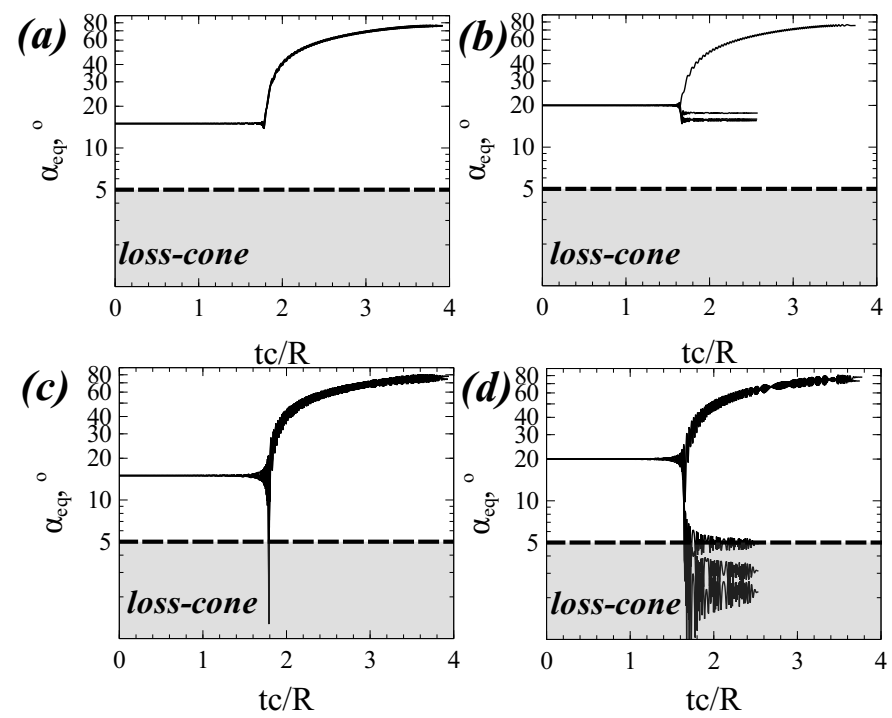

FIG. 7: Pitch-angle change along electron trajectories for $L=6$ (see details of the wave model in the caption of Fig. 1). Panels (a) and (b) show trajectories for $B_{w}=500 \mathrm{pT}$; panels (c) and (d) shows trajectories for $B_{w}=3 \mathrm{nT}$. Left and right panels show results for initial $\alpha=15^{\circ}$ and $\alpha=20^{\circ}$. The loss-cone is shown for illustration, $\alpha_{L C} \approx 5^{\circ}$.

strate that the nonlinear scattering of small pitch-angle electrons with $\Delta I_{x}>0$ does not necessary stop electron precipitations.

The pitch-angle jump $\Delta \alpha_{e q}$ is proportional to $\Delta I_{x}$ given by Eq. (22), and thus there is a scaling $\Delta \alpha_{e q} \sim$ $B_{w}^{2 / 3}$. If $\alpha_{e q}^{*}$ depends on $B_{w}$ sufficiently weakly, then the wave amplitude increase would result in $\left|\nu^{\prime}\right|$ increase. Therefore, for sufficiently high $B_{w}$ we should expect the regime with electron nonlinear scattering into loss-cone (see Fig. 6, left panels). To check this scenario, we integrate numerically set of electron trajectories given by Hamiltonian equations (2) for the same initial $\gamma$ and two wave amplitudes. Figure 7 shows that for large $B_{w}$ electrons can be scattered into loss-cone and precipitate.

\section{B. Two types of phase portraits}

We consider nonlinear resonant interaction of waves and electrons with small $I_{x}$, for which the resonant Hamiltonian takes the form given by Eq. (10) or Eq. (11). Let us compare this Hamiltonian with one describing the classical problem of charged particle scattering for moderate $I_{x}$ values (see Refs. 22 and 23). Instead of expansion of Hamiltonian (3) around $I=0$, we can expand it around the resonant value $I=I_{\text {res }}(s, P)$ determined from $\partial H / \partial I=0$ equation:

$$
\begin{aligned}
H & =-\omega I_{\text {res }}+\gamma_{\text {res }}+\frac{1}{2 m_{e}} K_{r e s}^{2}\left(I-I_{\text {res }}\right)^{2} \\
& +\sqrt{\frac{2 I_{r e s} \Omega_{c e}}{m_{e}}} \frac{e B_{w}}{c k \gamma_{r e s}} \sin \varphi
\end{aligned}
$$

where $K_{\text {res }}=\partial^{2} H /\left.\partial I^{2}\right|_{I=I_{\text {res }}}$ and $\gamma_{\text {res }}=\left.\gamma\right|_{I=I_{\text {res }}}$. The main difference of this Hamiltonian and one from Eq. (4) is that the effective wave amplitude does not depend on $I$ in Eq. (23). The phase portrait of Hamiltonian (23) with frozen slow variables is shown in Fig. 8(a).

Instead of introducing $(q, p)$ coordinates it is more convenient to introduce $P_{\phi}=I-I_{\text {res }}$ through the generation function $W=\left(I-I_{\text {res }}\right) \varphi+P s^{*}$ with new slow variables $s^{*}=s+\left(\partial I_{r e s} / \partial P\right) \varphi, P^{*}=P-\left(\partial I_{\text {res }} / \partial P\right) \varphi$. Expanding $I_{\text {res }}(s, P)$ and $\gamma_{\text {res }}(s, P)$ over $\left(\partial I_{\text {res }} / \partial P\right),\left(\partial I_{\text {res }} / \partial s\right)$, we rewrite Hamiltonian (23) as

$$
\begin{aligned}
H & \approx-\omega I_{\text {res }}+\gamma_{\text {res }} \\
& +\frac{K_{r e s}^{2} P_{\varphi}^{2}}{2 m_{e}}-\left\{\gamma_{r e s}, I_{\text {res }}\right\} \varphi+\sqrt{\frac{2 I_{r e s} \Omega_{c e}}{m_{e}}} \frac{e B_{w}}{c k \gamma_{r e s}} \sin \varphi
\end{aligned}
$$

where $\{\ldots\}$ are Poisson brackets, and all function in Eq. (24) depend on $\left(s^{*}, P^{*}\right)$. The phase portrait of Hamiltonian $(24)$ with the frozen slow variables $\left(s^{*}, P^{*}\right)$ is shown in Fig. 8(b). This is the classical portrait of the pendulum with torque ${ }^{67}$ with three main phase space regions: before resonance $P_{\varphi}=0$ crossing particles are in $G_{\text {outer }}$, and resonance crossing can result in trapping (particles appear in $G_{\text {inter }}$ ) or scattering (particles appear in $G_{\text {inner }}$ ). Therefore, there is direct relation between three regions $G_{\text {outer,inner, inter }}$ of Hamiltonian of (11) and Hamiltonian (24).

For the initial system given by Eq. (3) nonlinear scattering (transition from $G_{\text {outer }}$ to $G_{\text {inner }}$ ) always appears with $I_{x}$ decrease, and this effect is well seen in the phase portrait (c) of Fig. 8: the area $S_{\text {outer }}$ is always larger than the area $S_{\text {inner }}$. But when the initial invariant $I_{x}$ $\left(I_{p}\right.$ or $\left.I_{\varphi}=(2 \pi)^{-1} \oint P_{\varphi} d \varphi\right)$ is sufficiently small, particles become trapped within region $G_{\text {inter }}$ as soon as this region appears during particle motion along their trajectories. In phase portrait (c) of Fig. 8 this trapping means that the area surrounded by the particle trajectory $2 \pi I_{p} \approx 2 \pi Y$ is smaller than $S_{\text {inter }}$ at the moment when $G_{\text {inter }}$ appears (when $y_{R}$ becomes larger than one, see Fig. 3(c)). For $y_{R}=1$ we get $\eta_{c}=0, \tilde{q}_{c}=1$, and $S_{\text {inter }}=4 \pi Y_{R}^{*}$. Thus, the threshold $I_{x}$ value is $2 I_{x} \Omega_{c e} / m_{e} c^{2}=3\left(\Omega_{c e} / k c\right)^{2}\left(B_{w} / \kappa B\right)^{2 / 3}$. 

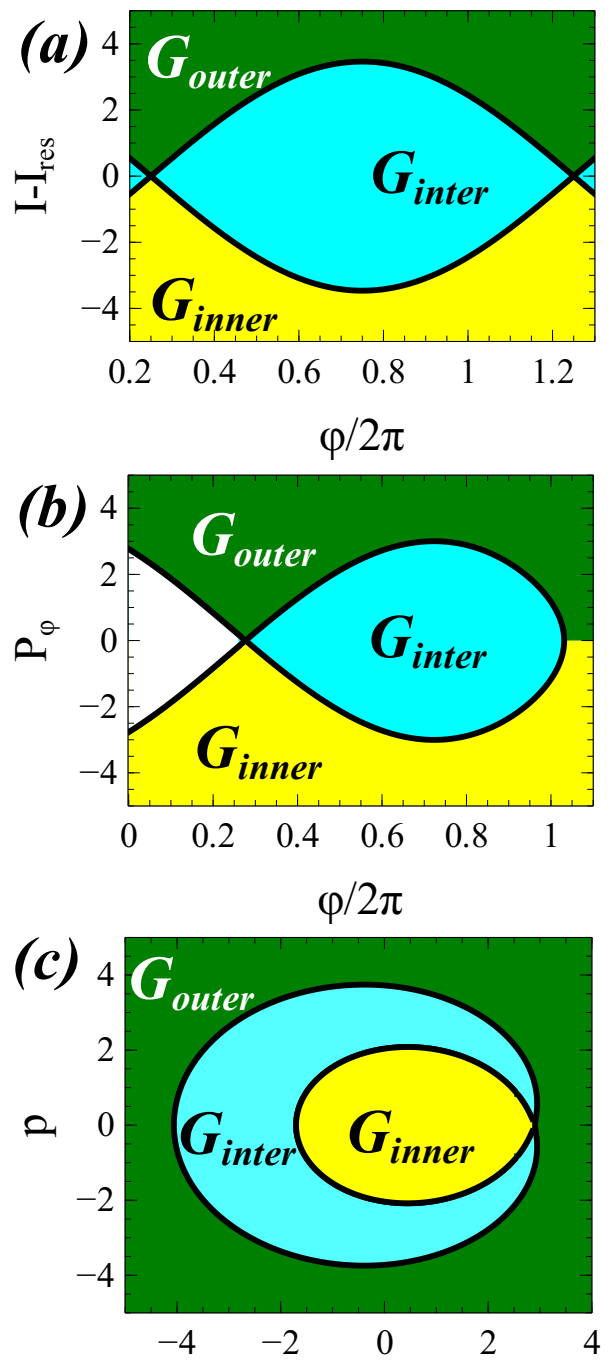

$\mathrm{q}$

FIG. 8: Phase portraits of Hamiltonian (23), panel (a), of Hamiltonian (24), panel (b), and Hamiltonian (11), panel (c).

This is so-called autoresonance phenomena of the $100 \%$ trapping in the resonant systems (see, e.g., Refs. 71-73 and references therein). Being trapped into $G_{i n t e r}$, particles can both increase or decrease their $I_{p}$ (and $I_{x}$ ) during the transition from $G_{i n t e r}$ to $G_{\text {inner }}$ : the $I_{x}$ change depends on the ratio of $S_{\text {inter }} / S_{\text {inner }}$ at the moment when $S_{\text {inter }}$ becomes equal to $2 \pi I_{p}$. For sufficiently small $I_{p}$ (small $I_{x}$ ), the ratio $S_{\text {inter }} / S_{\text {inner }}=2 \pi I_{p} / S_{\text {inter }}$ will be below one, and particles will increase their $I_{p}$ (and $I_{x}$ ) due to the resonant interaction. Formally, this interaction cannot be called scattering, because particles are trapped into $G_{\text {inter }}$ from the beginning.

\section{Small $I_{x}$ for electrons resonating with electrostatic waves}

For resonances in inhomogeneous plasma, there is the direct analogy between electron interaction with whistler-mode waves and with electrostatic waves ${ }^{15,74-77}$. Therefore, we can expect a change of the regime of electron nonlinear resonant interaction with electrostatic waves for field-aligned electrons. The main difference between the cyclotron resonance with electromagnetic waves (e.g., whistler-mode waves) and the Landau resonance with electrostatic waves is that for electrostatic waves the wave amplitude $U_{w}=e \Phi_{w}$ is determined by the electric field potential $\Phi_{w}$ and does not depend on $I_{x}$. Thus, Eqs. (7) would take the form:

$$
\dot{I}=-e \Phi_{w} \cos \varphi, \quad \dot{\varphi}=\frac{1}{m_{e}} K^{2}\left(I-I_{R}\right)
$$

Defining a small parameter $\varepsilon=e \Phi_{w} / m_{e} c^{2}$ (the analog of $e B_{w} / m_{e} c^{2} k_{e q}$ used through the paper), we can estimate the threshold value of $I_{x}$ for which there is no time separation of $I$ and $\varphi$ variations in Eqs. (25). For $\tau \sim t \varepsilon^{\beta}$ and $Y \sim I \varepsilon^{-\beta}$, Eqs. (25) give $\beta=1 / 2$. Thus, instead of the scaling $I_{x} \sim\left(e B_{w} / k\right)^{2 / 3}$ for the cyclotron resonance, we obtain the scaling $I_{x} \sim\left(e \Phi_{w}\right)^{1 / 2}$ for the Landau resonance.

Electrons can resonate with whistler mode waves through the Landau resonance, if waves are obliquely propagating, i.e. if there is a finite angle $\theta$ between a wavevector and a background magnetic field. For a general case of $\theta \neq 0$, the whistler-mode wave amplitude in Hamiltonian (3) takes the form ${ }^{47,56,78,79}$ :

$$
\begin{aligned}
U_{w} & =\sqrt{\frac{2 I_{x} \Omega_{c e}}{m_{e} c^{2}}} \frac{e B_{w}}{k} \sum_{ \pm} \frac{\cos \theta \pm C_{1}}{2 \gamma} J_{n \pm 1}\left(\sqrt{\frac{2 I_{x} k^{2}}{m_{e} \Omega_{c e}}} \sin \theta\right) \\
& +\frac{e B_{w}}{k}\left(\frac{p_{\|}}{\gamma m_{e} c}+C_{2}\right) J_{n}\left(\sqrt{\frac{2 I_{x} k^{2}}{m_{e} \Omega_{c e}}} \sin \theta\right) \sin \theta
\end{aligned}
$$

where $n$ is the resonance number, $C_{1,2}$ are functions of wave dispersion and $\theta$, and $J_{n}$ are Bessel functions. Equation (26) shows that for the cyclotron resonance $n=-1$ and small $I_{x}$ we have $U_{w} \sim \sqrt{I_{x}}$, and thus $I_{x} \sim\left(e B_{w} / k\right)^{2 / 3}$ scaling works. For the Landau resonance $n=0$ and small $I_{x}$ we have $U_{w} \sim \sin \theta \cdot$ (const + $\left.I_{x}\right) \sim O\left(I_{x}\right)$, and thus $I_{x} \sim\left(e B_{w} / k\right)^{1 / 2}$ scaling works.

\section{Model applicability and verification}

The proposed theoretical model predicts the parametrical boundary in the energy/pitch-angle space $\left(2 I_{x} \Omega_{c e} / m_{e} c^{2} \sim \varepsilon^{2 / 3}\right.$ or, more precise, $y_{R}=1$ in Eq. (20)) where the nonlinear scattering (phase bunching) disappears and the nonlinear wave-particle interaction is 
totally dominated by the phase trapping that transports resonant electrons away from the loss-cone. This parametrical boundary depends on wave and background filed characteristics, and can be evaluated for each specific plasma system. Thus, this boundary can be implemented into numerical models utilizing the generalized FokkerPlank equations ${ }^{39,56,80}$ and mapping techniques ${ }^{81}$. Such models require boundary conditions for the nonlinear wave-particle interaction domain (in the energy/pitchangle space), and our model shows that the low pitchangle boundary of this domain is the boundary of $100 \%$ of trapping.

The most straightforward verification of the derived theoretical mode consists in comparison of theoretical predictions with measurements of low-altitude spacecraft probing energetic electron precipitations from the Earth's radiation belts ${ }^{82-85}$. Such measurements, being combined with the equatorial measurements of wave characteristics, can provide the energy range of electrons trapped around loss-cone and not precipitating due to the effect $100 \%$ phase trapping.

\section{CONCLUSIONS}

In this study we propose the theoretical model of nonlinear resonant interaction of small pitch-angle electrons with whistler-mode waves propagating in the inhomogeneous magnetic field. Basic parameters and wave characteristics correspond to whistler-mode waves in the Earth's radiation belts. Using the adiabatic invariant $I_{x}$ (magnetic moment $\mu=e I_{x} / c$ ) and the small system parameter $\varepsilon \approx e B_{w} / m_{e} c^{2} k$ ), we show that

- The threshold value of $I_{x}$ for a new regime of nonlinear resonant electron transport away from the loss-cone is scaling with $\varepsilon$ as: $I_{x} \Omega_{c e} / m_{e} c^{2} \sim \varepsilon^{2 / 3}$. For $I_{x}$ smaller than this threshold value, the model of electron resonant interaction should include $\sim$ $\sqrt{I_{x}}$ factor for the wave amplitude.

- For $I_{x}$ below the threshold value, the $I_{x}$ change due to the phase trapping for all resonant electrons, whereas the precise system dynamics is described by theory of separatrix crossing given by Refs. 60 and 61 .

We also should note that the proposed theoretical model describes the ideal system with the monochromatic coherent whistler-mode wave. A wave field modulation is known to influence significantly the nonlinear resonant interaction ${ }^{41-43,70,86-88}$. Thus, to understand relevance of the proposed model to the electron resonant interaction with whistler-mode waves in the Earth's radiation belts, a more systematic numerical investigation of electron resonances for realistic short wave-packets whistlermode waves $^{29,89}$ is needed.

\section{ACKNOWLEDGMENTS}

A.V.A. are thankful to M. Kitahara and Y. Katoh for pointing on the problem of the nonlinear resonant interaction of small pitch-angle electrons ${ }^{50}$, to V. Grach and A. Demekhov for very useful discussion of their results on EMIC waves scattering $52,53,70$, to D. Shklyar for discussion of initial research of this problem ${ }^{49}$, and to D. Mourenas for useful suggestions. Work of A.V.A. and A.I.N. was supported by the Russian Scientific Foundation, project 19-12-00313. A.I.N. also was supported by the Leverhulme Trust, project RPG2018-143. W.L. and Q.M. would like to acknowledge the NASA grants 80NSSC19K0845, 80NSSC20K0698, 80NSSC20K0196, and NSF grants AGS-1723588 and AGS-1847818. L.G. would like to thank the NASA FINESST grant 80NSSC20K1506.

\section{DATA AVAILABILITY}

This is theoretical study, and all figures are plotted using numerical solutions of equations provided with the paper. The data used for figures and findings in this study are available from the corresponding author upon reasonable request.

${ }^{1}$ A. A. Galeev and R. Z. Sagdeev, "Nonlinear Plasma Theory," in Reviews of Plasma Physics, Volume 7, Reviews of Plasma Physics, Vol. 7, edited by A. M. A. Leontovich (1979) p. 1.

${ }^{2}$ A. A. Vedenov and R. Z. Sagdeev, "Some properties of a plasma with an anisotropic ion velocity distribution in a magnetic field," in Plasma Physics and the Problem of Controlled Thermonuclear Reactions, Volume 3, edited by M. A. Leontovich (1961) p. 332.

${ }^{3}$ A. A. Andronov and V. Y. Trakhtengerts, Geomagnetism and Aeronomy 4, 233-242 (1964).

${ }^{4}$ C. F. Kennel and H. E. Petschek, J. Geophys. Res. 71, 1-28 (1966).

${ }^{5}$ R. M. Thorne, Geophys. Res. Lett. 372, L22107 (2010).

${ }^{6}$ R. M. Millan and R. M. Thorne, Journal of Atmospheric and Solar-Terrestrial Physics 69, 362-377 (2007).

${ }^{7}$ W. Li and M. K. Hudson, Journal of Geophysical Research (Space Physics) 124, 8319-8351 (2019).

${ }^{8}$ W. E. Drummond and D. Pines, Nuclear Fusion Suppl. 3, 1049 1058 (1962).

${ }^{9}$ A. A. Vedenov, E. Velikhov, and R. Sagdeev, Nuclear Fusion Suppl. 2, 465-475 (1962).

${ }^{10}$ V. D. Shapiro and R. Z. Sagdeev, Physics Reports 283, 49-71 (1997).

${ }^{11}$ V. I. Karpman, Space Sci. Rev. 16, 361-388 (1974).

${ }^{12}$ D. Le Queau and A. Roux, Solar Physics 111, 59-80 (1987).

${ }^{13}$ J. M. Albert, J. Geophys. Res. 106, 8477-8482 (2001).

14 J. M. Albert, J. Geophys. Res. 115, A00F05 (2010).

${ }^{15}$ D. Nunn, Journal of Plasma Physics 6, 291 (1971).

${ }^{16}$ D. Nunn, Plan. Sp. Sci. 22, 349-378 (1974).

${ }^{17}$ V. I. Karpman, J. N. Istomin, and D. R. Shklyar, Plasma Physics 16, 685-703 (1974).

${ }^{18}$ V. I. Karpman, I. N. Istomin, and D. R. Shkliar, Physica Scripta 11, 278-284 (1975).

${ }^{19}$ A. I. Neishtadt and A. V. Timofeev, Sov. Phys. JETP 66, 973 (1987).

${ }^{20}$ M. D. Tokman and E. V. Suvorov, Sov. J. Plasma Phys. 14, 557-561 (1988).

${ }^{21}$ Y. Omura, H. Matsumoto, D. Nunn, and M. J. Rycroft, Journal of Atmospheric and Terrestrial Physics 53, 351-368 (1991). 
${ }^{22}$ D. R. Shklyar and H. Matsumoto, Surveys in Geophysics 30 55-104 (2009).

${ }^{23}$ J. M. Albert, X. Tao, and J. Bortnik, "Aspects of Nonlinear Wave-Particle Interactions," in Dynamics of the Earth's Radiation Belts and Inner Magnetosphere, American Geophysical Union, edited by D. Summers, I. U. Mann, D. N. Baker, and M. Schulz (2013).

${ }^{24}$ A. V. Artemyev, A. I. Neishtadt, D. L. Vainchtein, A. A. Vasiliev, I. Y. Vasko, and L. M. Zelenyi, Communications in Nonlinear Science and Numerical Simulations 65, 111-160 (2018).

${ }^{25}$ A. V. Artemyev, O. Agapitov, D. Mourenas, V. Krasnoselskikh, V. Shastun, and F. Mozer, Space Sci. Rev. 200, 261-355 (2016).

${ }^{26}$ L. B. Wilson, III, C. A. Cattell, P. J. Kellogg, J. R. Wygant, K. Goetz, A. Breneman, and K. Kersten, Geophys. Res. Lett. 38, L17107 (2011).

${ }^{27}$ X. J. Zhang, R. Thorne, A. Artemyev, D. Mourenas, V. Angelopoulos, J. Bortnik, C. A. Kletzing, W. S. Kurth, and G. B. Hospodarsky, Journal of Geophysical Research (Space Physics) 123, 5379-5393 (2018).

${ }^{28}$ E. Tyler, A. Breneman, C. Cattell, J. Wygant, S. Thaller, and D. Malaspina, Geophys. Res. Lett. 46, 2328-2336 (2019).

${ }^{29}$ X. J. Zhang, D. Mourenas, A. V. Artemyev, V. Angelopoulos, J. Bortnik, R. M. Thorne, W. S. Kurth, C. A. Kletzing, and G. B. Hospodarsky, Geophys. Res. Lett. 46, 7182-7190 (2019).

${ }^{30}$ Y. Omura, N. Furuya, and D. Summers, J. Geophys. Res. 112, A06236 (2007).

${ }^{31}$ D. Summers and Y. Omura, Geophys. Res. Lett. 34, L24205 (2007).

${ }^{32}$ N. Furuya, Y. Omura, and D. Summers, "Relativistic turning acceleration of radiation belt electrons by whistler mode chorus," J. Geophys. Res. 113, A04224 (2008)

${ }^{33}$ J. M. Albert, J. Geophys. Res. 105, 21191 (2000).

${ }^{34}$ A. V. Artemyev, A. A. Vasiliev, D. Mourenas, A. I. Neishtadt, O. V. Agapitov, and V. Krasnoselskikh, Physics of Plasmas 22, 112903 (2015).

${ }^{35}$ A. G. Demekhov, V. Y. Trakhtengerts, M. J. Rycroft, and D. Nunn, Geomagnetism and Aeronomy 46, 711-716 (2006).

${ }^{36}$ A. G. Demekhov, V. Y. Trakhtengerts, M. Rycroft, and D. Nunn, Geomagnetism and Aeronomy 49, 24-29 (2009).

${ }^{37}$ Y. Katoh and Y. Omura, Geophys. Res. Lett. 34, L13102 (2007).

${ }^{38}$ Y.-K. Hsieh and Y. Omura, J. Geophys. Res. 122, 675-694 (2017)

${ }^{39}$ Y. Kubota and Y. Omura, Journal of Geophysical Research (Space Physics) 123, 4835-4857 (2018).

${ }^{40}$ X. Tao, J. Bortnik, J. M. Albert, K. Liu, and R. M. Thorne, Geophys. Res. Lett. 38, L06105 (2011).

${ }^{41}$ X. Tao, J. Bortnik, J. M. Albert, R. M. Thorne, and W. Li, Journal of Atmospheric and Solar-Terrestrial Physics 99, 67-72 (2013).

${ }^{42}$ L. Gan, W. Li, Q. Ma, J. M. Albert, A. V. Artemyev, and J. Bortnik, Geophys. Res. Lett. 47, e85987 (2020).

${ }^{43}$ R. Hiraga and Y. Omura, Earth, Planets, and Space 72, 21 (2020).

${ }^{44}$ T. F. Bell, J. Geophys. Res. 89, 905-918 (1984).

${ }^{45}$ D. R. Shklyar, Sov. Phys. JETP 53, 1197-1192 (1981).

${ }^{46}$ A. I. Neishtadt, B. A. Petrovichev, and A. A. Chernikov, Soviet Journal of Plasma Physics 15, 1021-1023 (1989).

${ }^{47}$ J. M. Albert, Physics of Fluids B 5, 2744-2750 (1993).

${ }^{48}$ V. V. Solovev and D. R. Shkliar, Sov. Phys. JETP 63, 272-277 (1986).

${ }^{49}$ B. V. Lundin and D. R. Shkliar, Geomagnetism and Aeronomy 17, 246-251 (1977).

${ }^{50}$ M. Kitahara and Y. Katoh, J. Geophys. Res. 124, 5568-5583 (2019).

${ }^{51}$ Y. Kubota and Y. Omura, Journal of Geophysical Research (Space Physics) 122, 293-309 (2017).

${ }^{52}$ V. S. Grach and A. G. Demekhov, Radiophysics and Quantum Electronics 60, 942-959 (2018).

${ }^{53}$ V. S. Grach and A. G. Demekhov, Radiophysics and Quantum Electronics 61, 389-401 (2018).
${ }^{54}$ D. Vainchtein, X.-J. Zhang, A. Artemyev, D. Mourenas, V. Angelopoulos, and R. M. Thorne, J. Geophys. Res. (2018), $10.1029 / 2018 \mathrm{ja0} 25654$

${ }^{55}$ T. H. Stix, The Theory of Plasma Waves, New York: McGrawHill, 1962 (1962).

${ }^{56}$ A. V. Artemyev, A. I. Neishtadt, A. A. Vasiliev, and D. Mourenas, Journal of Plasma Physics 84, 905840206 (2018).

${ }^{57}$ A. I. Neishtadt, Russian Mathematical Surveys 69, 771 (2014).

${ }^{58}$ C. Cattell, J. R. Wygant, K. Goetz, K. Kersten, P. J. Kellogg, T. von Rosenvinge, S. D. Bale, I. Roth, M. Temerin, M. K. Hudson, R. A. Mewaldt, M. Wiedenbeck, M. Maksimovic, R. Ergun, M. Acuna, and C. T. Russell, Geophys. Res. Lett. 35, L01105 (2008).

${ }^{59}$ O. V. Agapitov, A. Artemyev, V. Krasnoselskikh, Y. V. Khotyaintsev, D. Mourenas, H. Breuillard, M. Balikhin, and G. Rolland, J. Geophys. Res. 118, 3407-3420 (2013).

${ }^{60}$ A. Neishtadt, Journal of Applied Mathematics and Mechanics 39, 594-605 (1975).

${ }^{61}$ J. Henrard and A. Lemaitre, Celestial Mechanics 30, 197-218 (1983).

${ }^{62}$ L. D. Landau and E. M. Lifshitz, Vol. 1: Mechanics, 1st ed., Course of Theoretical Physics (Oxford: Pergamon Press, 1960).

${ }^{63}$ A. Neishtadt, Journal of Applied Mathematics and Mechanics 45, 58-63 (1981).

${ }^{64}$ A. I. Neishtadt, Soviet Journal of Plasma Physics 12, 568-573 (1986).

${ }^{65}$ J. R. Cary, D. F. Escande, and J. L. Tennyson, Physical Review A 34, 4256-4275 (1986).

${ }^{66}$ A. Neishtadt, Journal of Applied Mathematics and Mechanics 51, 586-592 (1987).

${ }^{67}$ V. I. Arnold, V. V. Kozlov, and A. I. Neishtadt, Mathematical Aspects of Classical and Celestial Mechanics, 3rd ed., Dynamical Systems III. Encyclopedia of Mathematical Sciences (SpringerVerlag, New York, 2006).

${ }^{68}$ A. Neishtadt, Nonlinearity 32, R53-R76 (2019).

${ }^{69}$ J. M. Albert and J. Bortnik, Geophys. Res. Lett. 36, L12110 (2009).

${ }^{70}$ V. S. Grach and A. G. Demekhov, Journal of Geophysical Research (Space Physics) 125, e27358 (2020).

${ }^{71} \mathrm{~J}$. Fajans and L. Frièdland, American Journal of Physics 69 1096-1102 (2001).

${ }^{72}$ L. Friedland, Scholarpedia 4, 5473 (2009).

${ }^{73}$ A. I. Neishtadt, A. A. Vasiliev, and A. V. Artemyev, Regular and Chaotic Dynamics 18, 686-696 (2013), arXiv:1309.6501 [nlin.CD].

${ }^{74}$ S. P. Gary, D. Montgomery, and D. W. Swift, J. Geophys. Res. 73, 7524-7525 (1968).

${ }^{75}$ G. Laval and R. Pellat, J. Geophys. Res. 75, 3255-3256 (1970).

${ }^{76}$ V. I. Karpman and D. R. Shklyar, Sov. JETP 35, 500 (1972).

${ }^{77}$ V. I. Karpman, J. N. Istomin, and D. R. Shklyar, Physics Letters A 53, 101-102 (1975).

${ }^{78} \mathrm{X}$. Tao and J. Bortnik, Nonlinear Processes in Geophysics 17, 599-604 (2010).

${ }^{79}$ D. Nunn and Y. Omura, J. Geophys. Res. 120, 2890-2911 (2015).

${ }^{80}$ Y. Omura, Y. Miyashita, M. Yoshikawa, D. Summers, M. Hikishima, Y. Ebihara, and Y. Kubota, J. Geophys. Res. 120, 9545-9562 (2015).

${ }^{81}$ A. V. Artemyev, A. I. Neishtadt, and A. A. Vasiliev, Physics of Plasmas 27, 042902 (2020), arXiv:1911.11459 [physics.plasm-ph].

${ }^{82} \mathrm{~W}$. Li, D. Mourenas, A. Artemyev, O. Agapitov, J. Bortnik, J. Albert, R. M. Thorne, B. Ni, C. A. Kletzing, W. S. Kurth, and G. B. Hospodarsky, Geophys. Res. Lett. 41, 6063-6070 (2014).

${ }^{83}$ A. B. Crew, H. E. Spence, J. B. Blake, D. M. Klumpar, B. A. Larsen, T. P. O'Brien, S. Driscoll, M. Handley, J. Legere, S. Longworth, K. Mashburn, E. Mosleh, N. Ryhajlo, S. Smith, L. Springer, and M. Widholm, Journal of Geophysical Research (Space Physics) 121, 5272-5283 (2016).

${ }^{84}$ A. W. Breneman, A. Crew, J. Sample, D. Klumpar, A. Johnson, O. Agapitov, M. Shumko, D. L. Turner, O. Santolik, J. R. 
Wygant, C. A. Cattell, S. Thaller, B. Blake, H. Spence, and C. A. Kletzing, Geophys. Res. Lett. 44, 11,265-11,272 (2017).

${ }^{85}$ V. Angelopoulos, E. Tsai, L. Bingley, C. Shaffer, D. L. Turner, A. Runov, W. Li, J. Liu, A. V. Artemyev, X. J. Zhang, R. J. Strangeway, R. E. Wirz, Y. Y. Shprits, V. A. Sergeev, R. P. Caron, M. Chung, P. Cruce, W. Greer, E. Grimes, K. Hector, M. J. Lawson, D. Leneman, E. V. Masongsong, C. L. Russell, C. Wilkins, D. Hinkley, J. B. Blake, N. Adair, M. Allen, M. Anderson, M. Arreola-Zamora, J. Artinger, J. Asher, D. Branchevsky, M. R. Capitelli, R. Castro, G. Chao, N. Chung, M. Cliffe, K. Colton, C. Costello, D. Depe, B. W. Domae, S. Eldin, L. Fitzgibbon, A. Flemming, I. Fox, D. M. Frederick, A. Gilbert, A. Gildemeister, A. Gonzalez, B. Hesford, S. Jha, N. Kang, J. King, R. Krieger, K. Lian, J. Mao, E. McKinney, J. P. Miller, A. Norris, M. Nuesca, A. Palla, E. S. Y. Park, C. E.
Pedersen, Z. Qu, R. Rozario, E. Rye, R. Seaton, A. Subramanian, S. R. Sundin, A. Tan, W. Turner, A. J. Villegas, M. Wasden, G. Wing, C. Wong, E. Xie, S. Yamamoto, R. Yap, A. Zarifian, and G. Y. Zhang, "The ELFIN Mission," Space Sci. Rev. 216, 103 (2020), arXiv:2006.07747 [physics.space-ph].

${ }^{86}$ D. Nunn, Plan. Sp. Sci. 34, 429-451 (1986).

${ }^{87}$ A. V. Artemyev, D. Mourenas, O. V. Agapitov, D. L. Vainchtein, F. S. Mozer, and V. V. Krasnoselskikh, Physics of Plasmas 22, 082901 (2015).

${ }^{88}$ D. Mourenas, X.-J. Zhang, A. V. Artemyev, V. Angelopoulos, R. M. Thorne, J. Bortnik, A. I. Neishtadt, and A. A. Vasiliev, J. Geophys. Res. 123, 4979-4999 (2018).

${ }^{89}$ X. J. Zhang, D. Mourenas, A. V. Artemyev, V. Angelopoulos, W. S. Kurth, C. A. Kletzing, and G. B. Hospodarsky, Geophys. Res. Lett. 47, e88853 (2020). 